\title{
10 años de fomento a la investigación científica en la UNAH
}

Fernando José Zorto Aguilera ${ }^{1}$

\section{Introducción}

En la Universidad Nacional Autónoma de Honduras existe un movimiento de cambio en la forma de hacer investigación. Este cambio en la forma de hacer investigación. Este cambio radical se gesta desde hace diez (10) años, cuando la Junta de Transición Universitaria genera la cuarta reforma universitaria teniendo como eje primordial el ingreso a los temas de investigación científica. Por tal motivo, este ensayo se orienta hacia el eje de la política actual de la investigación científica, desarrollo tecnológico e innovación dentro de la Universidad, que es el fomento de la misma investigación en todos los niveles y estructuras en la Educación Superior.

El ensayo se encuentra constituido por 4 secciones. La primera hablará sobre la investigación científica universitaria, la importancia de ella dentro de los países y su papel en el desarrollo económico de toda nación. En la segunda, se plantearán las políticas de la UNAH, según las cuales, obedeciendo el mandato de la Ley Orgánica de la Universidad, es necesario visualizarlas para ver como el fomento a la investigación es un proceso fundamental para el crecimiento de la producción científica de la Universidad desde hace más de 10 años. En la tercera sección se plantean todos los avances en el fomento de la investigación dentro de la Universidad; esto lleva al lector a la cuarta sección, que habla sobre los retos que tiene que afrontar la Universidad para seguir con el éxito parcial que ha llevado hasta este momento. En su apartado final se realiza la discusión del tema mostrando que se ha hecho mucho en el fomento, pero no hay que bajar la guardia para seguir siendo una Universidad pionera a nivel nacional en los temas de investigación científica.

\section{La investigación científica universitaria}

La Educación Superior de toda nación cumple con el principio básico de la enseñanza de profesiones intelectuales, la investigación científica y la preparación de futuros investigadores. Es importante visualizarla desde la perspectiva de Ortega y Gasset (1930), sobre la Misión de la universidad. Él mencionaba que en esta institución, los jóvenes adquieren las capacidades y competencias básicas para convertirse en un

\footnotetext{
1 Profesor auxiliar, Departamento de Ingeniería Mecánica, Facultad de Ingeniería, UNAH: fernando.-
} zorto@unah.edu.hn 
médico, farmacéutico, licenciados, ingenieros, etc. Sin embargo, en ella ocurre una función muy importante para el país: cultivar la ciencia dentro de todas las instancias de la vida universitaria; así dicho autor argumentó que "dentro de la universidad se investiga y se enseña a ello".

La investigación científica se convierte en una herramienta para buscar un problema y luego entenderlo. Este entendimiento surge de la creación de una idea creativa y original, requisito altamente sensible entre los científicos. Una persona distraída del problema es poco probable que logré desarrollar una idea que haga un cambio radical a nuestra realidad. Mientras que los científicos desde su visión del campo de conocimiento buscan una perspectiva amplia del problema para transformar la realidad del mundo (Wilson, 1990).

Thomas Kunn (1962) indica que esta transformación es el cambio de paradigma, el cual se busca para incrementar el conocimiento de la propia realidad. Kunn manifestó que la ciencia es una constelación de conocimientos realizados por hombres que se han esforzado en la contribución de algún otro elemento en la constelación.

Karl Popper (1962) menciona que la ciencia es el conocimiento que nos ayuda a entender nuestra realidad a través de sistemas teóricos que sufren un constante cambio, dado que diversos hombres de todo el mundo continúan experimentando y descubriendo enfoques de cómo comprender la naturaleza del universo y del comportamiento humano.

Esto significa que la realidad de una nación puede cambiar de manera más rápida si existe un proceso de inversión real en la investigación científica. Es posible analizar las estadísticas que tienen las naciones más importantes económicamente, las cuales invierten en investigación a millares, dando como principio que la investigación genera múltiples ventajas competitivas en comparación a otras naciones, logrando tener el poder del mercado. En el sitio web de SCIMAGO se logra visualizar a las cincos naciones más poderosas del mundo: Estados Unidos, China, Reino Unido, Alemania y Japón, las cuales puntean como desarrolladores de conocimiento. Es importante mencionar que la producción cientíica no bajó de los 2 millones de artículos científicos durante un periodo de 20 años.

Ellos saben que el incremento del conocimiento científico dentro su nación los posiciona en una ventaja competitiva en comparación con otras naciones que no producen este tipo de conocimiento. Significa que las naciones del tercer mundo se encuentran en un desconocimiento de su propia realidad y cómo combatir sus problemas. 
Los socios de estas naciones para este proceso científico deberían ser las universidades, las cuales invierten altas sumas de dinero para generar el campo fértil para la investigación universitaria. Al respecto, Miguel Sánchez (2004) indica que la investigación requiere del reconocimiento de la cultura científica de Krebs, esto quiere decir que es imperativo que investigadores formen investigadores, por lo cual es importante disponer de maestros adecuados y buenos alumnos para establecer una cátedra de investigación que pueda hilvanar los factores de disciplina, rigurosidad científica y moralmente comprometida con la universidad. Logrando que las líneas y los programas de investigación continúen en la posteridad a beneficios de las naciones.

Por tal motivo, Sánchez (2004) considera que la investigación científica es un pilar fundamental de una nación, pero requiere del sacrificio financiero para su realización y difusión de resultados, como también la formación y especialización de los investigadores. Pero, no solo es de capacitar personas, sino adecuarlas con la infraestructura, equipos y la remuneración necesaria para el desarrollo de una carrera científica a lo largo de los años.

En las universidades centroamericanas el tema de la investigación científica ha tomado un movimiento algo lento, pero se está arrancando en esta carrera por el conocimiento. Honduras se posiciona en el puesto número 5 , con la Universidad Nacional Autónoma de Honduras (UNAH), como una de las mejores universidades a nivel regional, lo que resulta de la contundente reforma universitaria que ha realizado en los últimos 10 años (QS Univesity ranking, 2016), en torno a su prestigio de egresados, calidad científica y publicaciones.

\section{Las políticas de investigación de la UNAH}

La UNAH, en su Ley Orgánica, Capítulo I, artículo 3, índice 1, 2 y 3; expresa claramente que los objetivos de la Universidad Nacional Autónoma de Honduras son formar profesionales de alto nivel académico y promover la investigación científica dentro de las áreas del conocimiento. La Universidad, para estar acorde con las nuevas exigencias de este nuevo milenio, tuvo que hacer frente y revalorar algunos aspectos importantes de las reformas anteriores que habían ocurrido dentro de la UNAH. Por tal motivo, en el año 2000, la comunidad universitaria buscó un proceso reformista en consonancia con la época, que la guiará hacia la transformación administrativa y académica para lograr un mejoramiento incremental en la educación superior en calidad, pertinencia y equidad.

La cuarta reforma es el resultado de este movimiento, cuyo objetivo es lograr la 
producción científica, tecnológica y cultural de la UNAH para generar un proceso de cambio real en la República de Honduras (UNAH, 2009). Este mandato se encuentra dirigido desde la visión en donde la investigación sea un proceso orientado a la solución de problemas reales relacionados con el día a día de la nación, el cual debe estar inmerso en los diferentes niveles y estructuras de formación. Por lo cual, la investigación debe observarse como un proceso evolutivo que a lo largo de los años deberá ir creciendo a partir del reconocimiento de la realidad conocida y el entendimiento de la realidad desconocida.

Para analizar el proceso de investigación científica dentro de la Universidad, es necesario que se expliquen de manera general las políticas de investigación científica, desarrollo tecnológico e innovación $(I+D+i)$ de la UNAH, dado que ellas son los lineamientos a seguir para desarrollar eficazmente la creación y producción científica de la UNAH, cuyo objetivo general es el siguiente: "Impulsar, fortalecer y consolidar la investigación científica, tecnológica y la gestión de la misma, para contribuir al desarrollo de la ciencia y la tecnología y a la solución de los problemas nacionales, regionales e internacionales" (UNAH, 2014,p. 7).

En este punto es importante preguntar qué ámbitos de actuación tiene estas políticas de investigación, si son únicamente a lo interno de la UNAH o se extenderá de manera mucho más amplia fuera de los muros de la UNAH. Con respecto a la primera pregunta, la UNAH en las políticas de I+D+i tiene dos ámbitos o sitios de interacción: a lo interno de ella y luego en un plano externo, siendo una caja de resonancia para el apoyo de desarrollo tecnológico y científico a nivel nacional, regional e internacional.

Dentro de la UNAH es imperativo, para que se logre la implementación de dichas políticas, la socialización del trabajo integral de la investigación dentro de los programas académicos en los diferentes niveles y estructuras en la Educación Superior; siendo estas los programas de grados, posgrado u otras instancias que se encuentren directamente relacionadas con la investigación, tales como: institutos de investigación, consejos de investigación, grupos o redes de investigación y observatorios especializados adscritos a la carreras de grado y posgrado.

En el aspecto externo, las políticas de la UNAH ayudarán a posicionarla como socio estratégico de la sociedad hondureña e internacional para resolver problemas de índole nacional, regional e internacional; por lo que el objetivo de este ámbito es crear conocimiento que le sirva a la humanidad a conocer su universo. 
Para poder gestar las políticas de investigación, la Universidad plantea cinco ejes orientadores de la investigación, en los cuales a lo largo de 10 años se ha venido trabajando en ellos a pasos muy alargados, en comparación con los años anteriores, la cual fue una etapa muy oscura para la UNAH. Estos ejes son los siguientes: fomento a la investigación; publicación, difusión y comunicación; protección de los resultados de investigación, capacitación en investigación y en gestión de la investigación y gestión de la investigación.

\section{Fomento a la investigación, eje orientador}

El ensayo se centra en este eje primordial, según el cual las políticas de I+D+i de la UNAH (2014, p.14): "Condensa una serie de actividades de promoción cuya finalidad es reconocer, estimular la I+D+i, con el objetivo de formar este proceso de manera permanente". Dentro de las acciones de fomento a la investigación están las siguientes: becas de investigación, investigaciones sobre temas especiales, formación de investigadores en temas prioritarios de investigación y premios de investigación científica.

A lo largo de estos diez años de fomento de la investigación, la UNAH ha entregado 233 becas de investigación, las cuales se traducen en L.40, 499,255.09 invertidos para el desarrollo de conocimiento científico. El tema prioritario de investigación con mayor apoyo ha sido el de Salud, Estado y Sociedad, con un monto de L.14, 835,629.24. En el gráfico 1 se muestra el monto de becas otorgadas por la UNAH en las diferentes categorías.

Gráfico 1. Inversión en becas de investigación científica según tipo y presupuesto, UNAH, 2007-2015

Monto en lempiras

\section{L31053,458.09}

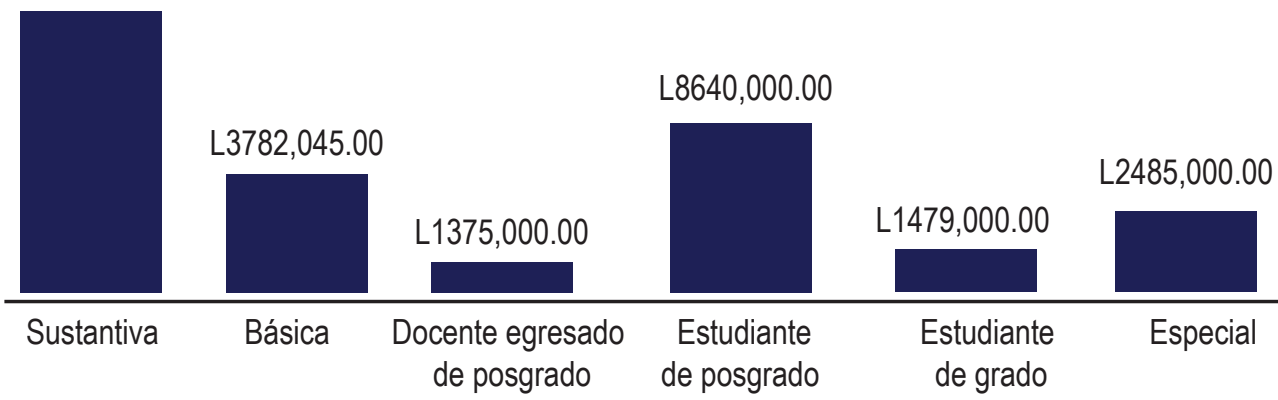

Fuente: Dirección de Investigación Científica y Posgrado. 
Hay que reconocer que los profesores de la Universidad o de las facultades como tal, concursan para becas sustantivas. Asimismo, es alentador que los profesores y estudiantes de posgrado consideren importante ser parte de estos montos de investigación. Pero, queda la duda si a los estudiantes y profesores de grado les interesa cultivar la investigación, es decir, desarrollarse como investigadores, dado que el $6 \%$ de las becas otorgadas fueron para estudiantes de grado.

Para poder optar a una beca, los profesores y estudiantes deben enmarcarse en los ejes de investigación, los cuales son: desarrollo económico y social, democracias y gobernabilidad, población y condiciones de vida, ambiente, biodiversidad y desarroIlo. El eje de población y condiciones de vida es el de mayor preferencia para los investigadores de la UNAH. El gráfico 2 muestra los diferentes ejes y la cantidad dinero invertido dentro de este proceso.

\section{Gráfico 2. Montos en lempiras invertidos en investigación científica por eje según presupuesto UNAH, 2007-2015}

Monto en lempiras

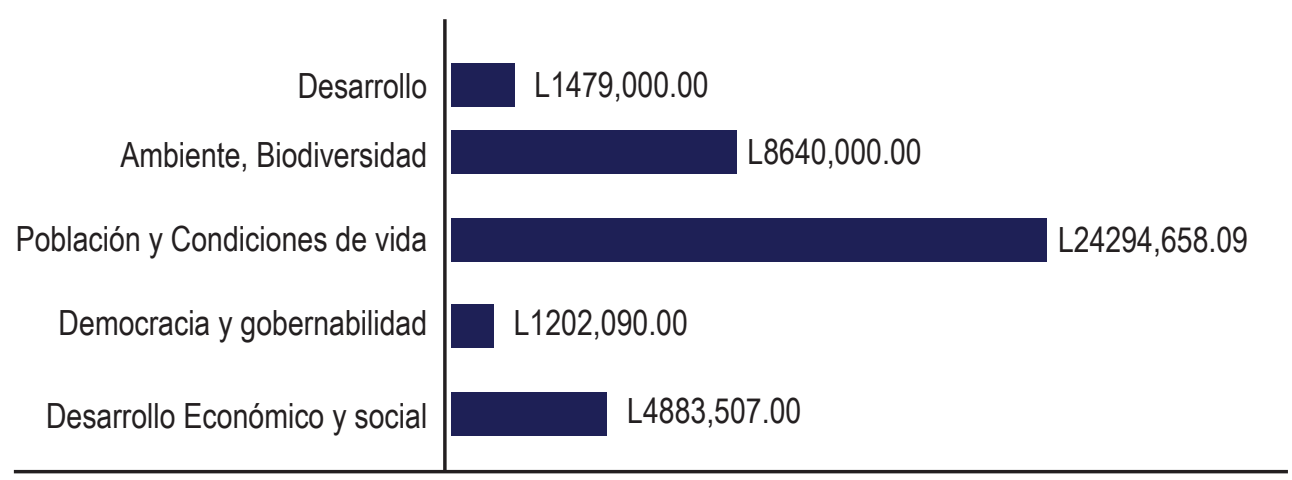

Fuente: Dirección de Investigación Científica y Posgrado.

Es importante recalcar que la Facultad de Ciencias se lleva la mayor cantidad de becas y fondos de investigación en comparación con otras unidades académicas, así como de los centros regionales. Dentro de estas becas se encuentran las becas básicas, sustantivas y especiales; de las cuales la Facultad de Ciencias ha logrado catorce (14) becas básicas, veintiséis (26) becas sustantivas y dos (2) especiales, por un valor total de L.13, 326,629.24; estas fueron otorgadas a personal docente de las diferentes carreras y unidades de investigación. Mientras tanto, los estudiantes de esta Facultad lograron una (1) beca de grado y catorce (14) de posgrado, con un monto total de L.615, 200.00. En contraposición, la Facultad de Odontología y el 
Centro Regional UNAH VS son las unidades académicas con menor producción científica, logrando cada una solamente una (1) beca de investigación en 10 años.

Como parte de este fomento a la investigación, la UNAH ha buscado mecanismos para que los profesores retornen a la práctica de investigación como parte de su actividad universitaria. Por este motivo, se inició en 2007 el otorgamiento de distinciones en investigación científica a personas destacadas del medio universitario, quienes por su cuenta y su constante participación en los procesos de investigación han puesto en alto el nombre de la Universidad. Así, se han entregado un total de 34 premios en diferentes categorías, que van desde una investigación ejemplar, con una alta calidad científica, hasta premios enfocados en el desarrollo de ideas de innovación en áreas estratégicas del desarrollo tecnológico para la UNAH.

Es importante mencionar que no solamente existe este tipo de incentivos, sino que también se considera el proceso de horas asignadas para investigación académica, en donde el profesor sede horas de trabajo al frente de estudiantes, para desarrollar plenamente su actividad en investigación. Desde el 2012 al 2015, se han otorgado más de 25,030 horas como carga académica, lo que equivale a un monto de inversión de L.5, 518,130.12.

Nuevamente la Facultad de Ciencias y la Facultad de Ciencias Espaciales, son las que tienen mayor beneficio de este incentivo. Es prudente indicar que este proceso ocurre de manera voluntaria por parte de los profesores y no es algo obligatorio que impongan las autoridades de cada unidad académica.

\section{Nuevos retos}

Actualmente, la labor en investigación se ha centrado en ciertas facultades, las cuales tienen una carrera en investigación muy larga, específicamente como la Escuela de Microbiología, cuyos docentes tienen perfiles profesionales y académicos de muy alto nivel. Los centros regionales y otras facultades deben de motivar a sus profesores, estudiantes de postrado y grado, a concursar por estos recursos destinados a la investigación.

El reto por parte de la Universidad es ligar estos montos de dinero de manera más equitativa para el desarrollo de la cultura de investigación en cada unidad académica dentro de la UNAH. En las facultades o en centros regionales es difícil concretar, individualmente como catedráticos, proyectos de investigación interesantes, por lo que sería conveniente apoyarse en otras personas interesadas de diferentes áreas 
del conocimiento para desarrollar grupos o redes de investigación interdisciplinarias para el fomento de líneas o programas de investigación.

La investigación es para aquellas personas que tengan una visión futurista de las cosas, esta idea es algo difícil de instalarla dentro de la mente de aquellos profesores que únicamente a largo de los años consideran que su labor es tomar marcador y pizarra para delinear la réplica de conocimiento que año tras año se ha venido dando en las aulas universitarias.

Si los profesores están renuentes al proceso de investigación, los estudiantes de pregrado no logran desarrollarse plenamente dentro del engranaje de la investigación como investigadores, coinvestigadores 0 asistentes de investigación, para ir alineándolos a este modelo educativo. Este proceso debe ir ligado al retorno de la tesis de grado como requisito de graduación, dado que este espacio universitario logra generar una visión clara de cómo llevar una investigación científica y no como lo plantea un seminario de investigación, en donde solo se muestran las características de una investigación y la formulación algo tosca de un anteproyecto de investigación, el cual es totalmente inservible para las unidades académicas.

No obstante, estos cambios deben ser consensuados por todas las partes: autoridades universitarias, profesores y estudiantes dentro del Consejo Universitario para la gestación nuevamente de las tesis universitarias como fomento al proceso de la investigación en la Universidad.

\section{Discusión}

La investigación científica de la Universidad ha venido tomando forma de manera lenta y aislada, pero en estos últimos 10 años, podemos decir que realmente sí ha existido un verdadero interés de las autoridades universitarias para ejercer el derecho natural de la Universidad para empezar a crear ciencia. Sin embargo, algunos detractores del proceso podrían decir que en sus unidades académicas no ha llegado aún, por lo que es importante señalar que los profesores y estudiantes realmente, en muchos de los casos, se encuentran renuentes, porque la información para concursar se encuentra fácilmente dentro los sitios cibernéticos de la UNAH. ¿Será la falta de actitud que tienen que les permite llevarlos a generar conocimientos? Creo que es la gran razón de que las personas no quieren ser parte de este cambio. Pero, es totalmente falso que la Universidad no ha invertido o que no ha fomentado la investigación científica, lo cierto es que existe renuencia en diversas unidades académicas para ser parte de este cambio. 
La falta de actitud es el cáncer que tienen muchos centros regionales y facultades en la UNAH, esto hace difícil gestionar los montos de dineros para la investigación en su área disciplinar. Realmente muchos de nuestros profesores y estudiantes tienen la carencia de poder realizar adecuadamente la investigación, dado que desconocen qué significa. En tal sentido, es necesario recordar que los procesos formativos en el ámbito de la investigación científica son voluntarios, lo cual significa que solo se forma con un pequeño grupo de profesores que poseen la actitud para investigar.

La apertura generalizada de estos espacios de manera obligatoria sería una acción irremediable para que estas personas con pocas ganas de cambiar su estado de confort puedan generar una nueva actitud positiva hacia el tema de la investigación científica. De esta forma, considero que los fomentos en investigación científica deberían ir orientados hacia aquellas unidades académicas apáticas al proceso de cambio de su rol formador, para pasar a un rol más activo en el desarrollo de la ciencia y del proceso formativo en el área de conocimiento en donde interactúan.

Todavía, a pesar de los logros de estos últimos 10 años, la Universidad tiene un largo camino que recorrer para seguir fomentado la investigación científica en cada uno de los miembros de la comunidad universitaria del país. Definitivamente se ha hecho mucho, pero en comparación con otros países falta bastante por avanzar, sin embargo, no podemos negar el éxito que hemos tenido hasta ahora si lo comparamos a como era antes.

\section{BIBLIOGRAFÍA}

Wilson, E. B. (1990). An Introduction to Scientific Research. New York, USA: Dover Publications, Inc.

Kuhn, T. (2004). Las Estructuras de la revoluciones Científicas. Mexico , México: Fondo de Cultura Económica .

Popper, K. (1962). La Logica de la Invetigación Cientifica. . Madrid: EDitorial Tecnos, S.A.

Sanchez, M. (2004). Editorial : Investigación Cientifica Universitaría. Revista Técnica de la Facultad de Ingeniería Universidad del Zulia, 27(1), 1-2.

Universidad Nacional Autónoma de Honduras. (2009). Modelo Educativa de la UNAH.

Ciudad Universitaría, Honduras: Editorial Universitaria.

Honduras, L. O. (2016). Dirección de Investigación cientifica y Postgrado. Recuperado el 23 de mayo de 2016, de Universidad Nacional Autónoma de Honduras: 
www.unah.edu.hn

Ortega y Gasset, J. (2007). Misión de la Universidad. Biblioteca nueva.

QS University Ranking . (23 de abril de 2016). QS Ranking: Latin America. Recuperado el 23 de junio de 2016, de QS Ranking Top Universities, Worldwide university rankins, guides \& events.: https://www.topuniversities.com

Scimago. (17 de julio de 2016). Scimago Journal \& Country Rank. Recuperado el 17 de julio de 2016, de Scimago: http://www.scimagojr.com/countryrank.php

UNAH. (2014). Politicas de Investigación cientifica, desarrollo tecnologico e innovación UNAH 2015-2019. Ciudad Universitaría, Honduras: Editorial Universitaria.

Wilson, E. B. (1990). An Introduction to Scientific Research. New York: Dover Publications. 